\title{
Factores de riesgo para la salud física en personas con enfermedad mental severa en México
}

\author{
Maribel Cruz-Ortiz,* Ma. del Carmen Pérez-Rodríguez, ** Cristina Jenaro-Río, ****María \\ Lourdes Hernández-Blanco, **** Noelia Flores-Robaina, *** Vanesa Vega Córdova ******
}

\begin{abstract}
RESUMEN
Se trata de un estudio descriptivo en una muestra por conveniencia de personas con enfermedad mental severa cuyo objetivo fue identificar factores de riesgo físico y necesidades de apoyo para el cuidado de la salud y la seguridad. Los principales factores de riesgo identificados en este estudio son el sobrepeso y la obesidad. La escasa identificación de los cuidadores primarios respecto a las necesidades de apoyo en el fomento de estilos de vida saludable puede aumentar la vulnerabilidad. La población estudiada constituye una población doblemente vulnerable a los factores de riesgo físico asociados a los estilos de vida. Esta vulnerabilidad está dada tanto por la evolución propia de la enfermedad mental como por la escasa integración de este grupo a los programas preventivos de los servicios de salud. El conocimiento de estos factores contribuye al diseño de políticas y programas que favorezcan la atención integral de este grupo.
\end{abstract}

Palabras clave: Factor de riesgo, estilo de vida, enfermedad mental severa.

\section{Risk factors for physical health in people with severe mental illness in Mexico}

\begin{abstract}
This is a descriptive study of a convenience sample of people with severe mental illness whose aim was to identify physical risk factors and support needs for health care and safety. The main risk factors identified in this study are overweight and obesity in people with severe mental illness, this coupled with the lack of identification of primary caregivers regarding the need for support in promoting healthy lifestyles can increase vulnerability. The study population is a population twice as vulnerable to physical risk factors associated with lifestyles. This vulnerability is due to both the typical evolution of mental illness as poor integration of this group to the preventive health services. Knowledge of these factors contributes to the design of policies and programs that promote comprehensive care of this group.
\end{abstract}

Key words: Risk factor, lifestyle, severe mental illness.

\footnotetext{
* Estudiante de Doctorado en Discapacidad en el Instituto de Integración en la Comunidad, Universidad de Salamanca, España.

** Doctora en Enfermería, San Luis Potosí, México. *** Doctora en Psicología, Universidad de Salamanca, España.

***** Licenciada en Enfermería, San Luis Potosí, México. ****** Estudiante de Doctorado, Universidad de Salamanca, España.
}

Correspondencia: Maribel Cruz Ortiz. Km. 8.5 Carretera a Matehuala, Soledad de Graciano Sánchez, San Luis Potosí, México.Teléfono (444) 83120 23, Correo electrónico: redazul@hotmail.com 


\section{Qué se conoce y qué aporta el artículo}

Las personas con enfermedades mentales tienen mayores tasas de enfermedad física y su esperanza de vida está reducida entre 13 y 20 años, comparada con cohortes no psiquiátricas; esto se relaciona con mayor prevalencia de riesgos por estilos de vida.

La medición de la intensidad de los apoyos que requieren estas personas respecto a salud y seguridad, proporcionados principalmente por los cuidadores primarios, es novedosa. Esta circunstancia tiene implicaciones en la planificación de servicios para ambos.

\section{INTRODUCCIÓN}

$\mathrm{L}$ as razones para considerar prioritaria la atención de personas con trastornos mentales y conductuales en términos de la salud pública se han centrado, en buena medida, en la magnitud del problema. Cifras como las que señalan que aproximadamente un $10 \%$ de la población adulta es afectada por estas enfermedades y alrededor del $20 \%$ de todos los pacientes atendidos por profesionales de atención primaria padecen uno o más trastornos mentales ${ }^{1}$ fundamentan esta preocupación.

Estas cifras cobran más fuerza aun con el análisis de las enfermedades mentales mediante indicadores compuestos como el de años de vida saludable ajustados por discapacidad o por muerte prematura. A través de estos indicadores se constata que en América Latina las afecciones psiquiátricas y neurológicas, que constituían en 1990 el 8.8\% de los años de vida perdidos ajustados por discapacidad (AVISA), ${ }^{2}$ ascendieron en 2004 a $23.5 \% .^{3}$

El uso de estos indicadores brinda, además, la posibilidad de entender las necesidades de salud de las personas con enfermedad mental bajo una perspectiva más amplia, lo que pone de relieve la necesidad de brindar una atención integral y diversificada, ya que este grupo de personas, además de ser afectadas por las características propias del trastorno que presentan (larga duración, temprana edad de inicio, alta prevalencia y escasos tratamientos eficaces), cursan por las mismas etapas del ciclo vital que cualquier otra persona y comparten riesgos semejantes.

Algunos estudios ${ }^{4,5}$ muestran que las personas con enfermedades mentales tienen mayores tasas de enfermedad física, comparadas con la población en general, y su esperanza de vida se reduce entre 13 y 20 años, en contraste con sus cohortes no psiquiátricas. Se ha demostrado que enfermedades como la esquizofrenia acortan la vida, pues la comorbilidad física representa el $60 \%$ de las muertes prematuras no relacionadas con el suicidio.

Estudios como el mostrado por Saravane et $\mathrm{al}^{6}$ señalan que el aumento de la morbimortalidad en personas con enfermedad mental se debe principalmente a la enfermedad cardiovascular prematura (infarto de miocardio, accidentes cerebrovasculares, etc.). Estos eventos cardiovasculares están estrechamente vinculados a factores de riesgo no modificables, como la edad, el género, antecedentes personales y/o familiares, pero también a factores cruciales de riesgo modificables, como el sobrepeso y la obesidad, la dislipidemia, la diabetes, la hipertensión, el abuso de sustancias y el tabaquismo, ${ }^{7}$ que pueden contribuir a su vez a la generación de discapacidades físicas asociadas a enfermedades médicas y que precisan de intervenciones adicionales, tanto para su prevención como para su control. ${ }^{7,8}$

Aunque estos factores de riesgo clásicos existen en la población en general, ${ }^{9}$ los estudios epidemiológicos sugieren que los pacientes con enfermedades mentales graves tienen una mayor prevalencia. Por ejemplo, algunos estudios señalan que estas personas tienen dos veces más propensión a fumar, y más de un $50 \%$ de ellos son propensos a tener sobrepeso u obesidad asociada a una mala alimentación e inactividad física. ${ }^{10}$

La interacción de todos los factores señalados hace patente que las personas con enfermedades mentales reúnen todas las condiciones para ser abordadas bajo una perspectiva más amplia, dado que presentan una vulnerabilidad mayor generada en buena medida por su falta de inclusión social y dificultades para acceder a los servicios de salud integrados y no sólo centrados en la atención psiquiátrica. ${ }^{11-13}$

Un elemento indispensable para el éxito en la atención a estas personas lo constituyen los apoyos brindados para ejercer a plenitud sus capacidades y particularmente en el área de la salud, para atender la prevención de riesgos. La medición de estas necesidades y de la intensidad con que se requieren ha sido poco abordada, de ahí que el objetivo del presente estudio sea identificar y describir la presencia de estos factores de riesgo para la salud física en personas con enfermedad mental severa en el contexto mexicano y el nivel de apoyo requerido en relación al área de salud y seguridad.

\section{MATERIAL Y MÉTODOS}

Se trata de un estudio descriptivo con muestreo por conveniencia, en el que se incluyó a personas con enfermedad mental severa que acudieron a la Consulta Externa de un hospital psiquiátrico público en México, con diagnóstico confirmado de enfermedad mental severa incluida en la clasificación CIE10; se buscó que la duración de dicha enfermedad fuera de más de dos años, con existencia de disfunción moderada o severa del funcionamiento global; que fueran mayores de 25 años y acudieran al Servicio de Consulta Externa al menos 2 veces durante el año 2009 en condiciones para la medición de la glucemia capilar y datos de somatometría. 
Una vez obtenido el consentimiento informado, los datos fueron recolectados entrevistando de forma conjunta al cuidador primario y al paciente en dos consultas programadas en las que se usó un cuestionario que agrupa variables demográficas (sexo, edad, estado civil, ocupación y escolaridad) y características clínicas (diagnóstico, duración de la enfermedad, frecuencia de la hospitalización, última hospitalización y duración de la misma). Todas ellas fueron categorizadas en respuestas de elección forzosa para homogeneizar los datos. La $2^{a}$ parte consistió en la obtención de los datos de somatometría y medición de las variables de tensión arterial y glucemia capilar obteniendo una cifra única en promedio para cada paciente. Por último, se recolectó información adicional a través de la subescala de actividades de salud y seguridad de la escala de intensidad de apoyos (SIS), que explora el apoyo que requiere el paciente en actividades relacionadas con los estilos de vida y el cuidado a la salud. Los datos se refieren a la necesidad de apoyo requerido para el cuidado de la salud de forma preventiva llevando a cabo conductas saludables (actividad física y cuidado de la salud), como conductas que eviten mayores daños (apego a tratamiento farmacológico). Estos ítems evalúan tres dimensiones: frecuencia, tiempo y tipo de apoyo requerido por la persona, en una escala de 0 a $4 .^{14}$

El análisis realizado es descriptivo para las variables sociodemográficas y clínicas; en el caso de los ítems de la subescala de salud y seguridad de la SIS, cada actividad está categorizada de acuerdo al baremo que posee la propia escala.

\section{RESULTADOS}

1) Datos sociodemográficos. Se obtuvieron datos de un total de 95 participantes con media de edad de 41.52 años (DT $=11.3$ ), de entre 25 y 72 años. El 63.2\% fueron varones (60), y el $36.8 \%$ mujeres (35); predominantemente resultaron solteros $(69.5 \%)$ y se encuentran inactivos en su mayoría $(67.4 \%)$.

2) Datos clínicos. El $89.47 \%$ tenía diagnóstico médico de esquizofrenia paranoide crónica y puntuación de funcionamiento global promedio de 54.25. Presentaron además media de 12.36 años de evolución con media de 1.55 internamientos hospitalarios [rango 1-6] aunque un $28.42 \%$ del total no ha tenido ninguno. El lapso de tiempo desde el último internamiento es de 2 a 5 años en un $17.89 \%$ de los casos. La duración del último internamiento se concentró entre 3 y 4 semanas en un $35.78 \%$ de la muestra y en segundo término en lapsos superiores a un año en un $27.36 \%$.

3) Datos de salud y seguridad. Las cuatro actividades analizadas en el cuadro I están relacionadas con estilos de vida y coinciden en agrupar los porcentajes mayores en el nivel mínimo de frecuencia de apoyo requerido, que corresponde a nunca o menos de una vez al mes, siendo el apoyo para mantener una dieta nutritiva la actividad señalada por los cuidadores primarios como la que menor frecuencia de apoyo requiere (43.15\%), mientras que la actividad que demanda mayor frecuencia de apoyo es la toma de medicación, en la que se refiere que un $33.68 \%$ de los pacientes requieren apoyo con una frecuencia de al menos una vez al día.

$\mathrm{Al}$ analizar las mismas actividades respecto al tiempo diario que el cuidador primario invierte en brindar apoyo (Cuadro II), podemos ver que también aquí el ítem de mantener una dieta nutritiva acumula el mayor porcentaje en el nivel mínimo de tiempo de apoyo, abarcando el $44.2 \%$ las personas que no lo requieren. En tanto, es la actividad de obtener servicios de cuidado para la salud la más demandante de tiempo, representando para el $46.3 \%$ de los cuidadores una inversión de tiempo de entre 30 minutos y 2 horas.

En concordancia con los dos parámetros anteriores (frecuencia y tiempo), el mayor porcentaje respecto al tipo de apoyo requerido para las 4 actividades evaluadas en conductas de salud y seguridad corresponde a ninguno (Cuadro III). Sin embargo, es interesante observar que el $2^{\circ}$ mayor porcentaje se acumula para las 4 actividades en ayuda física parcial, es decir, implica que el cuidador primario realice parte de la actividad con el paciente o bien le indique paso a paso cómo desarrollarla.

Cuadro I. Frecuencia de apoyo requerido en el Área de Salud y Seguridad.

\begin{tabular}{|c|c|c|c|c|c|c|c|c|}
\hline \multirow[t]{2}{*}{$\begin{array}{c}\text { Actividad } \\
\text { (frecuencia) }\end{array}$} & \multicolumn{2}{|c|}{$\begin{array}{c}\text { Tomar la } \\
\text { medicación }\end{array}$} & \multicolumn{2}{|c|}{$\begin{array}{l}\text { Obtener servicios de } \\
\text { cuidado a salud }\end{array}$} & \multicolumn{2}{|c|}{$\begin{array}{l}\text { Mantener una } \\
\text { dieta nutritiva }\end{array}$} & \multicolumn{2}{|c|}{$\begin{array}{l}\text { Mantener la salud y } \\
\text { buena forma física }\end{array}$} \\
\hline & No. & $\%$ & No. & $\%$ & No. & $\%$ & No. & $\%$ \\
\hline $\begin{array}{l}\text { Nunca o menos } \\
\text { de } 1 \text { vez al mes }\end{array}$ & 33 & 34,73 & 38 & 40 & 41 & 43.15 & 33 & 34,73 \\
\hline $\begin{array}{l}\text { Al menos una } \\
\text { vez al mes }\end{array}$ & 15 & 15,78 & 31 & 32,63 & 12 & 12,63 & 19 & 20,0 \\
\hline $\begin{array}{l}\text { Al menos una } \\
\text { vez a la semana }\end{array}$ & 15 & 15,78 & 23 & 24,21 & 26 & 27,36 & 30 & 31,57 \\
\hline $\begin{array}{l}\text { Al menos una vez } \\
\text { cada día }\end{array}$ & 32 & 33.68 & 3 & 3,15 & 16 & 16,84 & 13 & 13,68 \\
\hline
\end{tabular}


Se incluyeron dos actividades más en las Áreas de Salud y Seguridad, relacionadas con el tipo de apoyo requerido para prevenir el consumo excesivo de alcohol, tabaco y drogas, y mantener los tratamientos de la salud (Cuadro IV). Respecto a la primera actividad, el $29.6 \%$ de las personas de la muestra requieren algún apoyo o apoyo extenso. En concreto, el 23.15 tiene necesidades de apoyo relacionadas con el consumo de tabaco, variando en cantidades mínimas de 6 cigarrillos por día a 24 cigarrillos como máximo.
Por último, el ítem relacionado con el mantenimiento de tratamientos de la salud abarca las necesidades de apoyo para asistir a las citas y cumplir con el tratamiento, destacando que un $25.3 \%$ requiere apoyo extenso, es decir, dependen en gran medida o totalmente del familiar para desarrollar esta actividad (Cuadro IV).

4) Mediciones clínicas (Cuadro V). En todos los participantes se identificaron 3 factores de riesgo, pero en el caso de la medición de glucemia capilar se analizaron

Cuadro II. Tiempo de apoyo requerido en el área de salud y seguridad.

\begin{tabular}{|c|c|c|c|c|c|c|c|c|}
\hline \multirow[t]{2}{*}{$\begin{array}{l}\text { Actividad } \\
\text { (Tiempo) }\end{array}$} & \multicolumn{2}{|c|}{$\begin{array}{c}\text { Tomar la } \\
\text { medicación }\end{array}$} & \multicolumn{2}{|c|}{$\begin{array}{l}\text { Obtener servicios de } \\
\text { cuidado a la salud }\end{array}$} & \multicolumn{2}{|c|}{$\begin{array}{l}\text { Mantener una } \\
\text { dieta nutritiva }\end{array}$} & \multicolumn{2}{|c|}{$\begin{array}{l}\text { Mantener la salud y } \\
\text { buena forma física }\end{array}$} \\
\hline & No. & $\%$ & No. & $\%$ & No. & $\%$ & No. & $\%$ \\
\hline Nada & 33 & 34.7 & 37 & 38.9 & 42 & 44.2 & 33 & 34.7 \\
\hline $\begin{array}{l}\text { Menos de } 30 \\
\text { minutos }\end{array}$ & 22 & 23.2 & 14 & 14.7 & 10 & 10.5 & 17 & 17.9 \\
\hline $\begin{array}{l}\text { Más de 30'y } \\
\text { menos de } 2 \mathrm{~h}\end{array}$ & 34 & 35.8 & 44 & 46.3 & 38 & 40.0 & 38 & 40.0 \\
\hline Más de 2 h & 6 & 6.3 & & & 5 & 5.3 & 7 & 7.4 \\
\hline
\end{tabular}

Cuadro III. Tipo de apoyo requerido en el Área de Salud y Seguridad.

\begin{tabular}{|c|c|c|c|c|c|c|c|c|}
\hline \multirow[t]{2}{*}{$\begin{array}{l}\text { Actividad } \\
\text { (Tipo) }\end{array}$} & \multicolumn{2}{|c|}{$\begin{array}{c}\text { Tomar la } \\
\text { medicación }\end{array}$} & \multicolumn{2}{|c|}{$\begin{array}{l}\text { Obtener servicios de } \\
\text { cuidado a la salud }\end{array}$} & \multicolumn{2}{|c|}{$\begin{array}{l}\text { Mantener una } \\
\text { dieta nutritiva }\end{array}$} & \multicolumn{2}{|c|}{$\begin{array}{l}\text { Mantener la salud y } \\
\text { buena forma física }\end{array}$} \\
\hline & No. & $\%$ & No. & $\%$ & No. & $\%$ & No. & $\%$ \\
\hline Ninguno & 33 & 34.7 & 33 & 34.7 & 42 & 44.2 & 33 & 34.7 \\
\hline Supervisión & 19 & 20.0 & 19 & 20.0 & 8 & 8.4 & 13 & 13.7 \\
\hline $\begin{array}{l}\text { Incitación verbal } \\
\text { o gestual }\end{array}$ & 11 & 11.6 & 11 & 11.6 & 13 & 13.7 & 19 & 20.0 \\
\hline Ayuda física parcial & 25 & 26.3 & 25 & 26.3 & 30 & 31.6 & 26 & 27.4 \\
\hline Ayuda física total & 7 & 7.4 & 7 & 7.4 & 2 & 2.1 & 4 & 4.2 \\
\hline
\end{tabular}

Cuadro IV. Tipo de apoyo requerido en prevención de adicciones y control de la enfermedad.

\begin{tabular}{|c|c|c|c|c|}
\hline \multirow[t]{2}{*}{$\begin{array}{c}\text { Actividad } \\
\text { (Tipo de apoyo) }\end{array}$} & \multicolumn{2}{|c|}{$\begin{array}{l}\text { Prevención del abuso de alcohol, } \\
\text { tabaco y drogas }\end{array}$} & \multicolumn{2}{|c|}{$\begin{array}{l}\text { Mantenimiento de tratamientos } \\
\text { de salud mental }\end{array}$} \\
\hline & No. & $\%$ & No. & $\%$ \\
\hline No necesita apoyo & 66 & 69.5 & 41 & 43.2 \\
\hline Necesita algún apoyo & 22 & 23.2 & 30 & 31.6 \\
\hline Necesita apoyo extenso & 7 & 7.4 & 24 & 25.3 \\
\hline
\end{tabular}

Cuadro V. Variables clínicas.

\begin{tabular}{|c|c|c|c|c|}
\hline Variable & Parámetro & No. & $\%$ & $\begin{array}{c}\% \\
\text { acumulado }\end{array}$ \\
\hline Glucosa & Normal (- a $110 \mathrm{mg} / \mathrm{dL}$ en ayuno o = a 140 casual) & 41 & 73.21 & 73.21 \\
\hline \multirow[t]{2}{*}{$N=56$} & Sospecha (más de 120 mg/dL) & 7 & 12.5 & 85.71 \\
\hline & Confirmado cifra con diagnóstico confirmado) & 8 & 14.28 & 100.0 \\
\hline Tensión arterial & Normal (120-129/80-84 mm de Hg) & 69 & 72.6 & 72.6 \\
\hline \multirow[t]{3}{*}{$(\mathrm{N}=95)$} & Normal alta (130-139/85-89 mm de Hg) & 18 & 18.9 & 91.6 \\
\hline & Alta >140 mm de Hg (sistólica), y/o > 90 mm) & 6 & 6.3 & 97.9 \\
\hline & Diagnosticado & 2 & 2.1 & 100.0 \\
\hline IMC & Sobrepeso $(>25$ y $<27$ ) & 17 & 17.89 & 17.89 \\
\hline \multirow[t]{3}{*}{$(\mathrm{N}=95)$} & Obesidad $(>27)$ & 62 & 65.26 & 83.15 \\
\hline & Normal (entre 18 y 25$)$ & 13 & 13.68 & 96.83 \\
\hline & Bajo peso (menos de 18 ) & 3 & & 100.0 \\
\hline
\end{tabular}


sólo 56 casos (58.9\%), quienes reunieron las condiciones para efectuar las 2 mediciones requeridas, encontrando al $12.5 \%$ de ellas con cifras por encima de lo normal (más de $120 \mathrm{mg} / \mathrm{dL}$ en ayuno al promediar 2 tomas en días distintos), mientras que un $14.28 \%$ tenían ya un diagnóstico confirmado de diabetes mellitus y se encontraban en tratamiento, pero la mitad de ellas presentó cifras por encima de $200 \mathrm{mg} / \mathrm{dL}$.

Respecto a la variable tensión arterial, encontramos el mayor porcentaje de las personas evaluadas con cifras de entre 120-129/80-84 mm de $\mathrm{Hg}$ (72.6\%), considerada como normal, en tanto que el $25.2 \%$ presentan cifras de riesgo (normal alta o alta). ${ }^{15}$

Al analizar la variable índice de masa corporal (IMC), sólo un $13.68 \%$ presenta un peso recomendado; el $83.15 \%$ sobrepasa los 25 puntos de IMC, encontrándose con sobrepeso el $17.89 \%$ y con obesidad el $65.26 \%$, en proporciones semejantes para hombres y mujeres. El análisis por grupo de edad señala que el de mayor afectación en ambos sexos es el grupo de entre 36 y 45 años de edad, encontrándose con sobrepeso u obesidad el $31.6 \%$ de los hombres y el $28.6 \%$ de las mujeres.

Al revisar el acúmulo de factores de riesgo por persona, encontramos que un $7.36 \%$ de la muestra total $(\mathrm{N}=95)$, presentó cifras anormales tanto de glucemia capilar como de tensión arterial y un índice de masa corporal de sobrepeso u obesidad, mientras que un $26.31 \%$ presentó obesidad o sobrepeso y cifras anormales bien de presión arterial o de glucosa capilar, es decir, 2 factores de riesgo acumulados.

\section{CONCLUSIÓN}

Los factores de riesgo identificados en este estudio se encuentran entre aquellos que los Servicios de Salud analizan en la estimación de la contribución de factores de riesgo a la carga de mortalidad y morbilidad en la población adulta usando la evaluación comparativa de riesgo. La importancia de esta correspondencia radica en lo siguiente: 1) Probabilidad de encontrarse entre las principales causas de morbilidad en México, 2) Alta probabilidad de causalidad basada en la evidencia científica actual, 3) Ser potencialmente modificables (por ejemplo, si existen políticas que podrían reducir la exposición al factor de riesgo). ${ }^{17}$

El informe de la OMS sobre la Salud Mundial 2008, señala que una de las tendencias más preocupantes de los sistemas de salud es que están centrados en una oferta restringida de atención curativa especializada, pasando por alto las posibilidades que ofrecen las actividades de prevención primaria y promoción de la salud, con lo que se puede prevenir hasta el $70 \%$ de la carga de morbilidad. Al mismo tiempo, la atención primaria abre oportunidades a la prevención de enfermedades y la promoción de la salud, así como a la detección temprana de enfermedades, lo cual tiene implicaciones tanto para el paciente como para el cuidador primario, a la vista de la importancia que reviste su participación no sólo para brindar atención directa al paciente, sino también por la importancia de su participación en el fomento y adquisición de conductas saludables preventivas. ${ }^{16}$

Las cifras que hemos obtenido en las mediciones clínicas son interesantes por dos razones: encontramos niveles preocupantes en relación a variables clínicas como el IMC donde más del $80 \%$ de la población se encuentra por encima del nivel recomendado y la tensión arterial con un cuarto de los evaluados presenta cifras anormales (25.2\%), es decir, se identifica un riesgo real presente que con mucha frecuencia deriva de los estilos de vida. Este riesgo va acompañado de una baja percepción del mismo por parte del cuidador primario, reflejado en los bajos porcentajes obtenidos al interrogar sobre el apoyo que requiere el paciente para desarrollar actividades relacionadas con el cuidado de la salud y la prevención de riesgos.

Es importante señalar que los factores identificados se potencian y retroalimentan entre sí y pueden llevar a la aparición de enfermedades crónicas al sumarse a factores de probable riesgo como lo es el consumo prolongado de neurolépticos para el tratamiento psiquiátrico, en los que se recomienda precaución en su utilización, dado que se han encontrado asociados a problemas de aumento de peso, ${ }^{17}$ y descenso de glóbulos blancos en niños, adolescentes y ancianos. ${ }^{18}$

Por estas razones, se recomienda implementar medidas sencillas de control para personas con medicación antipsicótica tales como someterse a pruebas regulares de glucosa en sangre, perfil lipídico, medición específica de circunferencia de cintura para el control de peso corporal y control periódico de la presión arterial. También se recomienda acentuar la identificación de riesgo previo a la instalación del tratamiento e informar al paciente y su familia sobre los riesgos cardiovasculares y metabólicos. ${ }^{6,19-21}$

Como es posible observar, los planteamientos anteriores tienen varias implicaciones: en primer lugar, refuerzan la necesidad de integrar a las personas con enfermedades mentales a los programas preventivos normativos a los que accede la población en general, favoreciendo su inclusión social. En segundo término, enfatizan en la necesidad de estudios que caractericen la exposición a riesgos de este grupo en específico para tener mayores herramientas preventivas. Por último, en el estudio subyace la percepción que tienen los cuidadores primarios sobre la importancia del cuidado de la salud mediante la adopción de conductas saludables más allá de la atención de los síntomas. 
De todo lo anterior, se concluye que es necesario estudiar con más profundidad la intensidad de los apoyos que requieren estas personas en el área de la salud para diversificar los servicios, tomando como uno de sus ejes más importantes a los cuidadores primarios.

\section{BIBLIOGRAFÍA}

1. Marder SR, Essock SM, Miller AL, Buchanan RW, Casey DE, Davis JM et al. Physical health monitoring of patients with schizophrenia. 2004: 1334-49.

2. Kohn R, Levav I, Caldas de Almeida J, Vicente B, Andrade L, CaraveoAnduaga J, Saxena S, Saraceno B. Los trastornos mentales en América Latina y el Caribe: asunto prioritario para la salud pública. Rev Panam Salud Pública 2005; 18(4/5): 229-40.

3. WHO. Integrating mental health into primary care: A global perspective. Geneva: World Health Organization; 2008.

4. Lambert T, Velakoulis D, Pantelis C. Medical comorbidity in schizophrenia. The Medical Journal of Australia 2003; 178: 67-70.

5. Vreeland B. Bridging the gap between mental and physical health: a multidisciplinary approach. J Clin Psychiatry 2007; 68 (Suppl 4): 26-33.

6. Saravane D, Feve B, Frances Y, Corruble E, Lancon C, Chanson P, Maison P, Terra J, Azorin J. Drawing up guidelines for the attendance of physical health of patients with severe mental illness. Encephale. 2009; 35 (4): 330-9.

7. Dickey B, Normand S, Weiss R, Drake R, Azeni H. Medical morbidity, mental illness, and substance use disorders. Psychiatric Services 2002; 53 (7): 861-7.

8. Smith S, Yeomans D, Bushe Ch, Eriksson C, Harrison T, Holmes R, Mynors-Wallis L, Oatway H, Sullivan G. A well-being programme in severe mental illness. Reducing risk for physical ill-health: A post-programme service evaluation at 2 years. European Psychiatry 2007; 22 (7): 413-8.

9. Bradizza C, Maisto S. Predicting post-treatment-initiation alcohol use among patients with severe mental illness and alcohol use disorders. Journal of Consulting and Clinical Psychology 2009; 77 (6): 1147-58.

10. Compton M, Daumit G, Druss B. Cigarette smoking and overweight/ obesity among individuals with serious mental illnesses: a preventive perspective. Harv Rev Psychiatry 2006; 14 (4): 212-22.

11. Kessler R. Psychiatric epidemiology. Bulletin of the World Health Organization 2000; 78 (4): 464-74.

12. Murray C, López A. The global burden of disease: a comprehensive assessment of mortality and disability from diseases, injuries and risk factors in 1990 and projected to 2020. Cambridge: Harvard School of Public Health on behalf of the World Health Organization and the World Bank 1996.

13. Murray C, López A. Progress and directions in refining the global burden of disease approach: a response to Williams. Health Economy 2000; 9 (1): 69-82.

14. Verdugo M, Arias B, Ibáñez A. Escala de Intensidad de Apoyos. Manual (adaptación española del original: Supports Intensity Scale) 2007.

15. SSA. Norma Oficial Mexicana NOM-030-SSA2-1999, para la prevención, tratamiento y control de la hipertensión arterial. Secretaría de Salud 1999.

16. OMS. Informe sobre la salud en el mundo 2008: La atención primaria de salud, más necesaria que nunca. Ginebra: Organización Mundial de la Salud; 2008.

17. Komossa K, Rummel-Kluge C, Hunger H, Schmid F, Schwarz S, Duggan L, Kissling W, Leucht S. Olanzapine versus other atypical antipsychotics for schizophrenia. Cochrane Database Syst Rev 2010; 17(3).

18. Wahlbeck K, Cheine M, Essali MA. Clozapine versus typical neuroleptic medication for schizophrenia. Cochrane Database Syst Rev 2000; 2 .

19. Calkin C, van de Velde C, Růziková M, Slaney C, Garnham J, Hajek T, O'Donovan C, Alda M. Can body mass index help predict outcome in patients with bipolar disorder? Bipolar Disord 2009; 11 (6): 650-6.

20. McIntyre R, Konarski J, Misener V, Kennedy S. Bipolar disorder and diabetes mellitus: Epidemiology, etiology, and treatment implications. Ann Clin Psychiatry 2005; 17 (2): 83-93.

21. Newcomer J. Metabolic syndrome and mental illness. Am J Manag Care 2007; 13(Suppl 7): 170-7. 